\title{
The circadian gene Cryptochrome 2 influences stress-induced brain activity and depressive-like behavior in mice
}

\section{Sokolowska, Ewa}

2021-04

Sokolowska , E , Viitanen , R , Misiewicz , Z, Mennesson, M , Saarnio , S, Kulesskaya , N , Kängsep , S , Liljenback , H , Marjamäki , P , Autio , A, Callan , S-A , Nuutila , P , Roivainen , A , Partonen , T \& Hovatta , I 2021, ' The circadian gene Cryptochrome 2 influences stress-induced brain activity and depressive-like behavior in mice ', Genes, Brain and Behavior, vol. 20 , no. 4 , 12708 . https://doi.org/10.1111/gbb.12708

http://hdl.handle.net/10138/335381

https://doi.org/10.1111/gbb.12708

unspecified

acceptedVersion

Downloaded from Helda, University of Helsinki institutional repository.

This is an electronic reprint of the original article.

This reprint may differ from the original in pagination and typographic detail.

Please cite the original version. 


\section{The circadian gene Cryptochrome 2 influences stress-induced brain activity and depressive-like behavior in mice}

Ewa Sokolowska1 ${ }^{1}$, Riikka Viitanen ${ }^{2}$, Zuzanna M isiewicz ${ }^{1,3}$, M arie Mennesson ${ }^{1,3,4}$, Suvi Saarnio ${ }^{1}$, Natalia Kulesskaya ${ }^{1}$, Sanna Kängsep ${ }^{1}$, Heidi Liljenbäck ${ }^{2,5}$, Päivi M arjamäki ${ }^{2}$, Anu Autio ${ }^{2}$, Saija-Anita Callan ${ }^{1}$, Pirjo Nuutila ${ }^{2,6,7}$, Anne Roivainen ${ }^{2,5,7}$, Timo Partonen ${ }^{8}$, liris Hovatta ${ }^{1,3,4,8,9}$

${ }^{1}$ M olecular and Integrative Biosciences Research Program, University of Helsinki, Helsinki, Finland

${ }^{2}$ Turku PET Centre, University of Turku, Turku, Finland

${ }^{3}$ Department of Psychology and Logopedics, University of Helsinki, Helsinki, Finland

${ }^{4}$ SleepWell Research Program, Faculty of M edicine, University of Helsinki, Helsinki, Finland

${ }^{5}$ Turku Center for Disease M odeling, University of Turku, Turku, Finland

${ }^{6}$ Department of Endocrinology, Turku University Hospital, Turku, Finland

${ }^{7}$ Turku PET Centre, Turku University Hospital, Turku, Finland

${ }^{8}$ Department of Public Health Solutions, National Institute for Health and Welfare, Helsinki, Finland

${ }^{9}$ Neuroscience Center, Helsinki Institute of Life Science HiLIFE, University of Helsinki, Helsinki, Finland

Running title: Cry2 and behavior

Key words: circadian rhythm, cryptochrome, anxiety, depression, behavior, PET, gene expression, brain, brown adipose tissue

\section{Corresponding author:}

Prof. liris Hovatta

SleepWell Research Program

P.O. box 21

00014 University of Helsinki

Finland

Phone +358-50-4484-509

Email: iiris.hovatta@helsinki.fi

Date of submission: July 11, 2020, revised on September 15, 2020

\section{Number of words:}

Abstract 214

Introduction 860

Discussion 1337 


\section{ABSTRACT}

Cryptochrome 2 (Cry2) is a core clock gene important for circadian regulation. It has also been associated with anxiety and depressive-like behaviors in mice, but the previous findings have been conflicting in terms of the direction of the effect. To begin to elucidate the molecular mechanisms of this association, we carried out behavioral testing, PET imaging, and gene expression analysis of $\mathrm{Cry}^{-1-}$ and $\mathrm{Cry}^{+1+}$ mice. Compared to $\mathrm{Cry}_{2}{ }^{\mathrm{H}+}$ mice, we found that $\mathrm{Cry} 2^{-/-}$mice spent less time immobile in the forced swim test, suggesting reduced despair-like behavior. M oreover, Cry2 $2^{-1-}$ mice had lower saccharin preference, indicative of increased anhedonia. In contrast, we observed no group differences in anxiety-like behavior. The behavioral changes were accompanied by lower metabolic activity of the ventro-medial hypothalamus, suprachiasmatic nuclei, ventral tegmental area, anterior and medial striatum, substantia nigra, and habenula after cold stress as measured by PET imaging with a glucose analog. Although the expression of many depression-associated and metabolic genes was upregulated or downregulated by cold stress, we observed no differences between $\mathrm{Cry}^{-{ }^{--}}$and $\mathrm{Cry}_{2}{ }^{++}$mice. These findings are consistent with other studies showing that Cry2 is required for normal emotional behavior. Our findings confirm previous roles of Cry2 in behavior and extend them by showing that the effects on behavior may be mediated by changes in brain metabolism.

\section{INTRODUCTION}

Circadian clocks are endogenous pacemakers that evolve their properties, when subjected to selection, but have remained conserved during evolution ${ }^{1}$. They not only generate the oscillation in physiological functions and behaviors that demonstrate daily and seasonal fluctuations ${ }^{2}$, but also maintain these oscillations in their adaptive response to the time-giving signals which they receive from the habitat 3,4 . Circadian clock genes that encode repressors of transcription, or "the breaks" of the transcription-totranslation feedback loops, such as the cryptochrome genes (Cry1 and Cry2) ${ }^{5-9}$, are essential to the normal function of circadian clocks ${ }^{10,11}$. Cryptochromes are necessary for the development of intercellular networks in the suprachiasmatic nucleus ${ }^{12}$ that produces synchronizing signals throughout the organism ${ }^{3}$. 
Cryptochromes seem to regulate various behavioral traits, but the underlying mechanisms remain largely unknown. A specific mouse line selectively bred for high anxiety-like and co-segregating depression-like behaviors has fragmented ultradian rhythms of locomotor activity both under light-entrained conditions and under constant darkness, and reduced hippocampal Cry2 expression ${ }^{13}$. On the other hand, earlier studies on the behavior of cryptochrome knockout mice have yielded contradictory findings on anxiety-like and depression-like behaviors. De Bundel et al. ${ }^{14}$ found that Cry ${ }^{-1}$, Cry2 ${ }^{-1-}$ and Cry ${ }^{-1-C r y} 2^{-1-}$ double knockout mice have increased anxiety-like behavior in the elevated plus maze test and no differences in despair behavior in the forced swim test compared to controls. Similarly, Savalli et al. ${ }^{15}$ demonstrated increased anxiety-like behavior of Cry2 ${ }^{-/-}$mice in the novelty suppressed feeding test, but no differences in the open field, elevated plus maze or light-dark box tests compared to $\mathrm{Cry} 2^{++}$mice. Furthermore, they showed increased anhedonia in the sucrose preference test and no differences to $\mathrm{Cry2}^{\mathrm{H+}}$ mice in the forced swim or tail suspension tests for despair behavior. Hühne et al. ${ }^{16}$ observed that Cry ${ }^{-1-C r y 2}{ }^{-1-}$ double knockout mice have limited ability to habituate to new environments compared to wild type mice, but no differences in anxiety parameters of the open field or light dark box test, or in the tail suspension, learned helplessness or sucrose preference tests. When the Cry ${ }^{-/}$Cry $2^{--}$double knockout mice were exposed to an anxiogenic stressor, they had a larger number of c-Fos positive cells in the amygdala, suggesting increased neural activation compared to wild type mice. Schnell et al. ${ }^{17}$, however, found reduced anxiety-like behavior of $\mathrm{Cry}^{2-1-}$ mice compared to wild type mice in the elevated zero maze test and no anhedonia in the sucrose preference test. Cry $1^{-/}$mice had increased despair behavior in the forced swim test compared to wild type mice. Some of these differences may be due to genetic differences between the strains. De Bundel et al. ${ }^{14}$, Hühne et al. ${ }^{16}$, and Schnell et al. ${ }^{17}$ used a knockout line originally created by van der Horst et al. ${ }^{18}$, while Savalli et al. ${ }^{15}$ used a line created by Thresher et al. ${ }^{19}$ These lines were either backcrossed to C57BL/6J ${ }^{15,16}$ or "a Charles River C57BL/ 6 strain" ${ }^{14}$, or it was unclear whether the line was backcrossed ${ }^{17}$. Another difference between the studies was how knockout animals were generated, by either breeding heterozygous mice or by breeding the single knockouts with each other, in case of studies using double knockout mice. Knockout and heterozygous dams may provide different maternal care, thereby influencing 
the pup behavior. Thus, additional behavioral studies are needed to understand what the reasons for the differing results on anxiety and depressive-like behavior may be.

Under constant lighting conditions, with no light-dark transition, the knockout of both cryptochromes deletes the circadian rest-activity rhythm and makes the animals arrhythmic not only in their locomotor behavior ${ }^{18,20,21}$, but also in their body temperature and heat production ${ }^{22}$. Normal effective thermoregulation requires precise internal synchronization of the circadian clocks within the body ${ }^{23}$, with cryptochromes resisting against internal desynchronization in peripheral organs ${ }^{24}$. Brown adipose tissue (BAT) produces heat by uncoupling the mitochondrial respiratory chain with the tissue-specific uncoupling protein $1(\mathrm{UCP} 1)^{25}$, and this non-shivering thermogenesis can be activated by cold exposure ${ }^{26}$, and in hamsters and mice also by melatonin ${ }^{27}$. The activity of BAT follows a shorter than circadian (i.e., ultradian) period ${ }^{28}$ and precedes eating activity ${ }^{29}$. Further, it drives and guides the approximate 24 -hour circadian rhythm of the core body temperature 3,30 that is the anchor for all other circadian rhythms in the body and regulated against mild environmental cold exposures throughout the 24 -hour day ${ }^{23}$. In mice, BAT expresses Cry1 and Cry2 with a circadian rhythm ${ }^{31}$.

Thus, the CRY2 protein seems to modulate both circadian and emotional responses and metabolism. However, the cellular and molecular mechanisms connecting CRY2 function to these behaviors remain unknown. We therefore set to test anxiety and depression-related behaviors in Cry2 ${ }^{-1-}$ mice. Due to the central role of Cry2 in the metabolism, and the known associations between depression and metabolism ${ }^{32}$, we also investigated the metabolic activation of these mice in various tissues, including the brain and the BAT. After finding significant differences in the glucose uptake in specific brain regions of Cry2 ${ }^{-/-}$mice, we further examined the expression levels of key metabolic and depression-associated genes within the brain and BAT. We found that Cry2 is required for normal emotional behavior and brain metabolism.

\section{MATERIALS AND METHODS}

\section{Cry2 $^{-/-}$mice}


Cry2 ${ }^{-1-}$ mice (B6.129P2-Cry2 $\left.{ }^{\text {tm1Asn }} / \mathrm{J}\right)$ were obtained from the Jackson Laboratory (Bar Harbor, ME, USA). This strain was created by replacing flavine adenine dinucleotide (FAD) binding domain by a neomycin resistance cassette ${ }^{19} .129 \mathrm{P} 2 /$ OlaHsd-derived E14TG2a embryonic stem (ES) cells were used to create the mutation, which was subsequently backcrossed to the C57BL 6J strain. The strain was maintained at the University of Helsinki in mouse colony rooms under $12 \mathrm{~h}$ light/dark cycle (lights on at 6:00 am) in the M ouse IVC Green Line System (Tecniplast, West Chester, PA, USA) with a constant room temperature and humidity. M ice for all experiments were bred using Cry2 ${ }^{+-}$breeding pairs. We tested Cry $2^{-1-}$ mice and their Cry2 ${ }^{++}$littermates as controls.

\section{Behavioral testing}

All animal experiments were approved by the National Animal Experiment Board in Finland (ELLA) and the Regional State Administrative Agency for Southern Finland (ESAVI) and conducted in accordance with the European Union Directive. Adequate measures were taken to minimize pain or discomfort of the animals. M ice were housed in groups of 3-5 animals. Prior to any behavioral procedures, mice were transported from the mouse colony room to the experimental room and allowed to habituate for $30 \mathrm{~min}$. Testing was carried out during the light phase, between 8 am and 12 pm. If not specified, food and water were available ad libitum. Mice were 9-10 weeks old at the beginning of behavioral testing. We tested both male and female mice. The order of the tests and inter-test intervals were: the open-field test, 2 days later the lightdark box test, 4-5 days later the novelty-suppressed feeding test, and 12 days later the forced swim test (FST). The home cage activity test was carried out in three 7-day batches, the first one starting 2 days after the FST with equal number of Cry2 $2^{--}$and $\mathrm{Cry}_{2}{ }^{H+}$ mice in each batch. We carried out the saccharin preference test during the activity monitoring.

Open-field test. We released the mice in the corner of a novel open arena $(30 \times 30 \mathrm{~cm}$, Med Associates Inc., St. Albans, VT, USA) under indirect diffused light ( $150 \mathrm{~lx})$. Horizontal and vertical activity was recorded with infrared light sensors for $5 \mathrm{~min}$. Peripheral zone was defined as a $6 \mathrm{~cm}$ wide zone along the wall. 
Distance travelled, number of rearing events, and time spent in the two zones (center and periphery) were recorder by the Activity M onitor software (Med Associates Inc., St. Albans, VT, USA).

Light-dark box test was carried out in the open-field arena $(30 \times 30 \mathrm{~cm}$, M ed Associate Inc., St. Albans, VT, USA), equipped with a dark insert (nontransparent for visible light) that covered $50 \%$ of the arena. It was completely closed, except for a small opening $(5.5 \times 7 \mathrm{~cm})$ that allowed the animal to move freely from one compartment to another. Illumination in the center of the light compartment was $\sim 550$ Ix. We placed mice in the dark compartment and allowed them to explore the arena for $6 \mathrm{~min}$. The measures recorded by the Activity M onitor software were latency to enter the light compartment and the total time spent in the dark and light compartments. In addition, we measured the number of transitions between the compartments and the total distance travelled during the test.

Novelty-suppressed feeding test. The latency to start feeding in novel environment was measured by the EthoVisionXT10 software (Noldus Information Technology, Wageningen, Netherlands). Animals were deprived of food overnight before the experiment with water available ad libitum. For the measurement, we put small plastic cup containing chopped food pellets to the center of the floor in a white acrylic box (30 x $30 \mathrm{~cm}$, illumination $\sim 150 \mathrm{~lx}$ ). M ouse was placed at the corner of the box for 5 min or until the moment it started to eat the food. We then removed the mouse to the home cage and allowed it to eat the chopped food for the next $5 \mathrm{~min}$. The weight of food consumed for the $5 \mathrm{~min}$ in the home cage, as well as animals' body weight before and after the food deprivation were used as additional parameters in data analysis. Forced swim test. M ouse was placed in a glass cylinder $(\varnothing 18 \mathrm{~cm}$, height $25 \mathrm{~cm}$ ) filled with room temperature water to the height of $15 \mathrm{~cm}$ for $6 \mathrm{~min}$. The time of immobility (passive floating, when the animal was motionless or doing only slight movements with tail or one hind limb), and active movement (struggling, climbing or swimming using all four paws) was collected by EthoVisionXT10 software (Noldus, Information Technology, Wageningen, Netherlands), and analyzed in 2 min time bins.

Home cage activity. Mice were housed individually. An infrared InfraM ot (TSE Systems, Chesterfield, M O, USA) sensor was mounted above each cage to register animal movement. Ambient light changes as well as 
room temperature were recorded. Activity was registered for 7 consecutive days. Home cage activity was calculated as an average over $1 \mathrm{~h}$ observation. Days 1 and 2 of the experiment were treated as the habituation period and were not included in the statistical analysis.

Saccharin preference. Saccharin preference test was performed on the 1st-3rd night of the measurement of the home cage activity. On the night 1 we habituated the mice to drink water from two plastic $15-\mathrm{ml}$ falcon tubes with cut tips. On nights 2 and 3 mice had a free choice between tube with water and tube with $0.1 \%$ or $0.2 \%$ saccharin, respectively. The percentage of saccharin preference for each saccharin concentration was calculated from the weight of saccharin divided by the total weight of liquid (saccharin plus water) consumed by animal overnight.

Statistical analysis of the behavioral data. Open-field, light-dark box, novelty-suppressed feeding, and forced swim tests: We first examined any putative sex-effects with 2-way analysis of variance (ANOVA). Due to the lack of significant sex effects, the data from males and females were analyzed jointly with t-test or M ann-Whitney test (non-normally distributed data, normality tested by the Shapiro-Wilk test) in GraphPad (San Diego, CA, USA) Prism software. Saccharin preference: Three Cry2 ${ }^{H+}$ mice from the $0.1 \%$ group and one $\mathrm{Cry}^{-1-}$ mouse from the $0.2 \%$ group were removed from the analysis as outliers (saccharin preference $>3$ st.dev. from the mean), and thus it was not possible to analyze the data with 2-way ANOVA. We analyzed the two concentrations separately, comparing the genotypes using a t-test in GraphPad (San Diego, CA, USA) Prism software. Home cage activity was analyzed using IBM (Armonk, NY, USA) SPSS with 3way repeated measured ANOVA taking into account the activity level at each 1-h time bin, genotype, and sex.

\section{Cold exposure}

M ice were transferred to $4^{\circ} \mathrm{C}$ for $4 \mathrm{~h}$, with water and bedding but without food. Their body weight was measured before and after cold exposure. For the positron emission tomography/computed tomography (PET/CT) imaging, mice were individually transferred to smaller cages before putting them to $4^{\circ} \mathrm{C}$ to avoid them warming each other. For the RT-qPCR experiment, mice were singly housed for one week before the 
experiment to avoid the effect of social hierarchy on gene expression. For the RT-qPCR experiment control mice were housed in a room temperature incubator (Scantainer; Scanbur, Karlslunde, Denmark) during the same period.

\section{Body composition analysis}

Before the first room temperature and cold PET imaging, body composition was measured by quantitative nuclear magnetic resolution whole-body composition analyzer (EchoM RI-700, Echo M edical Systems, Houston, TX, USA). M ice were fasted for $4 \mathrm{~h}$ before measurement with ad libitum access to water. Awake mouse was restrained to a transparent cylinder tube and 2-3 measurements per animal were performed. The average fat tissue and lean tissue in grams was calculated. M ice were weighed before the measurement of body composition.

\section{PET/CT studies}

In vivo $\left[{ }^{18} \mathrm{~F}\right] \mathrm{FDG}$ PET/CT. Before PET imaging, mice were fasted for $4 \mathrm{~h}$ with ad libitum access to water. The PET/CT imaging was performed at room temperature with or without a preceding 4 -h cold exposure at $4{ }^{\circ} \mathrm{C}$. M ice were anesthetized with isoflurane, tail vein cannulated, and CT was performed for anatomical reference and for attenuation correction. Then, mice were intravenously injected with glucose analog 2deoxy-2-[18 F]fluoro-D-glucose ([18F]FDG, $5.1 \pm 0.27 \mathrm{M} \mathrm{Bq}$ ) or long-chain fatty acid analogue $14(\mathrm{R}, \mathrm{S})$ $\left[{ }^{18} \mathrm{~F}\right]$ fluoro-6-thia-heptadecanoic acid $\left(\left[{ }^{18} \mathrm{~F}\right] \mathrm{FTHA}, 5.3 \pm 0.29 \mathrm{M} \mathrm{Bq}\right)$ via tail vein. A 30-min dynamic PET imaging (Inveon Multimodality PET/CT scanner, Siemens Medical Solutions, Knoxville, TN, USA) started at the time of injection. Blood glucose levels were measured with a glucometer (Bayer Contour, Bayer AG, Leverkusen, Germany) before the $\left[{ }^{18} \mathrm{~F}\right] \mathrm{FDG}$ injection and after the $\left[{ }^{18} \mathrm{~F}\right] \mathrm{FDG}$ PET/CT imaging. In addition, blood samples were taken before $\left[{ }^{18} \mathrm{~F}\right] \mathrm{FTHA}$ injection to determine free fatty acid (FFA) levels in plasma. The PET data acquired in a list mode were iteratively reconstructed with the ordered subsets expectation maximization 2D algorithm. CT images were automatically fused with the PET images. Based on CT as the anatomical reference, quantitative PET analysis was performed by defining regions of interest (ROIs) in interscapular BAT and left, right, front and back brain, and to main organs using Carimas 2.6 software 
(Turku PET Centre, Turku, Finland). The average radioactivity concentration $(\mathrm{kBq} / \mathrm{mL})$ within the ROI was used for further analyses. The uptake of $\left[{ }^{18} \mathrm{~F}\right] \mathrm{FDG}$ is reported as standardized uptake values (SUV), which were calculated as the radioactivity concentration of the ROI normalized with the injected radioactivity dose, animal weight and blood glucose levels. Since reliable [ $\left.{ }^{18} \mathrm{~F}\right] \mathrm{FTHA}$ data was only observed from a few animals, only results from $\left[{ }^{18} \mathrm{~F}\right] \mathrm{FDG}$ imaging are presented. Group differences were analyzed with 3-way ANOVA for genotype, tissue, and temperature in IBM (Armonk, NY, USA) SPSS.

Density of brown adipose tissue. CT acquisition consisted of 120 projections with the exposure time of 120 $\mathrm{ms}$, x-ray voltage of $80 \mathrm{kV}$, and anode current of $210 \mu \mathrm{A}$ for $220^{\circ}$ rotation. CT images were reconstructed using filtered back-projection algorithm. Quantitative analysis was performed by defining ROI in BAT using Inveon Research Workplace 4.1 software (Siemens M edical Solutions, Malvern, PA, USA). CT Hounsfield units (HUs) were transformed to density as milligram per cubic centimeters $\left(\mathrm{mg} / \mathrm{cm}^{3}\right)$.

Ex vivo digital brain autoradiography. We sacrificed the animals directly after PET imaging, i.e. 30 min after the $\left[{ }^{18} \mathrm{~F}\right] \mathrm{FDG}$ injection. The brains were rapidly removed, weighed, measured for radioactivity using a gamma counter (Triathler 3", Hidex, Turku, Finland) and frozen in isopentane chilled with dry ice. Serial coronal brain sections $(20 \mu \mathrm{m})$ were obtained using a cryomicrotome at $-15^{\circ} \mathrm{C}$. Brain sections were thawmounted onto microscope slides, air dried and apposed to an imaging plate (Fuji Imaging Plate BAS-TR2025, Fuji Photo Film Co., Ltd., Japan) for autoradiography. After an exposure time of $4 \mathrm{~h}$, the imaging plates were scanned with Fuji Analyzer BAS-5000 (Fuji Photo Film Co., Ltd., Tokyo, Japan; internal resolution of $25 \mu \mathrm{m}$ ). The digital autoradiography images were analyzed for count densities (photostimulated luminescence per unit area, PSL $/ \mathrm{mm}^{2}$ ) using Aida software (Raytest Isotopenmessgeräte $\mathrm{GmbH}$, Straubenhardt, Germany). ROls were drawn over the studied brain regions and the count densities of background areas were subtracted from the image data.

Pieces from the cerebellar cortex of each brain were dissected, measured for ${ }^{18} \mathrm{~F}$-radioactivity and weighed. These data, expressed as the percentage of injected radioactivity dose per gram of tissue $(\% \mathrm{ID} / \mathrm{g})$, were used for converting the PSL/ $\mathrm{mm}^{2}$-values into \% $\mathrm{ID} / \mathrm{g}$-values and then into SUVs. For each individual brain 
region a mean uptake was calculated and expressed at a precision of one standard deviation (mean \pm SD). Group differences were analyzed with 2-way ANOVA for genotype and brain region in IBM (Armonk, NY, USA) SPSS. Post hoc test was carried out with multiple t tests option in GraphPad (San Diego, CA, USA) Prism.

\section{RNA extraction and quantitative real-time PCR}

M ice were killed by cervical dislocation. The brain was removed quickly and medial prefrontal cortex, hippocampus and hypothalamus were dissected on a Petri dish filled with ice, frozen immediately on dry ice, and stored at $-80^{\circ} \mathrm{C}$ for later analysis. Interscapular BAT was collected from the same animals. We extracted the total RNA using TRI reagent (M olecular Research Center, Inc., Cincinnati, OH, USA). cDNA was synthesized from 250 ng of RNA with BioRad iScript ${ }^{\text {mM }}$ Select cDNA Synthesis Kit (Bio-Rad Laboratories, Hercules, CA, USA) according to the manufacturer's protocols. 2-4 $\mu$ l of 1:10 dilution of the cDNA was amplified with 250 nM primers in CFX384 Real-Time PCR cycler using IQ SYBR Green supermix (Bio-Rad Laboratories, Hercules, CA, USA) in a total volume of $10 \mu$ l. Each reaction was run in triplicate and relative expression level was calculated with CFX M anager (Bio-Rad Laboratories, Hercules, CA, USA) using a standard curve $(7.15,10.0,5.0,2.0,1.0,0.5$, and $0.25 \mathrm{ng}$ of $\mathrm{CDNA})$ present on each assay plate. Expression levels were normalized to the levels of the endogenous control gene Cyclophilin A. The primer sequences are available from the authors on request. The significance of the observed gene expression differences was evaluated with 3-way ANOVA taking into account genotype, sex, and temperature. Post hoc analysis was carried out using Fisher's LSD test using IBM (Armonk, NY, USA) SPSS.

\section{RESULTS}

\section{Delayed activity during dark phase and altered depression-like behavior in Cry2 ${ }^{-/-}$mice}

To establish whether Cry2 regulates depression or anxiety-like behaviors we carried out extensive behavioral testing of littermate $\mathrm{Cry} 2^{-/}$and $\mathrm{Cry}_{2}{ }^{H+}$ mice. We did not observe any differences between these groups in anxiety-like behavior as measured by the time spent in the center zone of the open field test, the 
latency to the light side or the time spent in the light zone of the light-dark box test, or the latency to consume food in the novelty suppressed feeding test (Figure 1). Cry $2^{-1-}$ mice spent less time immobile in the forced swim test compared to Cry2 $2^{H+}$ mice, suggesting reduced despair-like behavior (Figure 2A). However, they had $2.2 \%$ and $1.2 \%$ lower saccharin preference (of $0.1 \%$ and $0.2 \%$ saccharin, respectively) compared to Cry $2^{++}$mice indicative of slightly increased anhedonia (Figure 2B). We also monitored home cage activity of $\mathrm{Cry2}^{-{ }^{-}}$and Cry2 ${ }^{+1+}$ mice (Figure 3). While the total activity between these groups did not differ (main effect for genotype $F 1,43=0.00, p=0.99)$, the activity* genotype interaction was significant $(F 23,989=7.69$, $\mathrm{p}=2.50 \mathrm{E}-23)$, suggesting that $\mathrm{Cry} 2^{-/-}$and $\mathrm{Cry}^{\mathrm{H+}}$ mice had different circadian activity patterns. Graphs in Figures $3 \mathrm{~B}$ and $\mathrm{C}$ show that this difference was due to Cry $2^{-1-}$ mice having about $2 \mathrm{~h}$ delayed onset of the first dark period activity peak compared to $\mathrm{Cry} 2^{+1+}$ mice. The activity*sex interaction was also highly significant $F 23,989=3.28, p=3.53 E-7$, indicating that the activity of males and females differed from each other.

\section{Lower brain metabolic activation in specific brain regions of Cry2 ${ }^{-/-}$mice}

To establish whether there are differences in the metabolic activity between $\mathrm{Cry}_{2}{ }^{--}$and $\mathrm{Cry} 2^{H+}$ mice, we carried out PET imaging with a glucose analog $\left(\left[{ }^{18} \mathrm{~F}\right] \mathrm{FDG}\right)$ and a fatty acid $\left(\left[{ }^{18} \mathrm{~F}\right] \mathrm{FTHA}\right)$ analog in room temperature and after 2-hour cold exposure, which activates the BAT. Due to the large number of failed $\left.{ }^{[18} \mathrm{F}\right] \mathrm{FTHA}$ measurements, we only present data using the $\left[{ }^{18} \mathrm{~F}\right] \mathrm{FDG}$ analog. We first verified that the cold exposure indeed influenced the BAT glucose consumption (SUV $\mathrm{glu}_{\text {; }}$ see Supplemental Figure 1 for plasma glucose levels of $\mathrm{Cry2}^{-\%}$ and $\mathrm{Cry} 2^{H+}$ mice). In the $\mathrm{Cry2}^{H+}$ mice, the BAT SUV glu was $17.3 \%$ higher after cold exposure compared to room temperature, indicating successful activation of BAT due to the cold exposure. We observed no difference in the BAT SUV glu between $\mathrm{Cry}^{-1-}$ and Cry2 ${ }^{+1+}$ mice (2-way repeated measures ANOVA for genotype and temperature: genotype effect $F 1,15=0.97, p=0.34$, temperature effect $F 1,15=14.68, p=0.002$, temperature* genotype interaction $F 1,15=4.26, p=0.057$ ).

We next proceeded to examine $\mathrm{SUV}_{\text {glu }}$ in all studied brain regions and both temperatures in $\mathrm{Cry}^{-{ }^{-1}}$ and Cry2 $2^{++}$mice. 3-way ANOVA of SUV glu for genotype, tissue, and temperature showed a highly significant 
main effect of the tissue (F8,27=267.82, $p=1.20 \mathrm{E}-123)$, indicating different $\mathrm{SUV}_{\text {glu }}$ in different tissues (Figure 4). Temperature (main effect $F 1,270=0.37, p=0.55$ ) or genotype (main effect $F 1,270=3.74, p=0.054$ ) did not

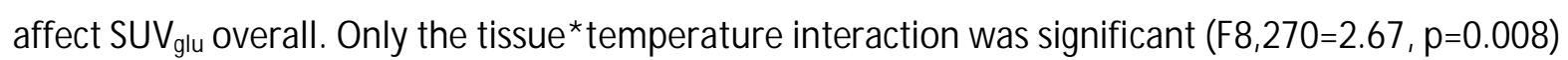
indicating that cold stress increased SUV $\mathrm{V}_{\text {glu }}$ in some, while decreased it in other tissues (genotype*tissue interaction: $F 8,270=0.56, p=0.81$; genotype*temperature $F 1,270=1.21, p=0.27$ ); genotype*tissue*temperature $\mathrm{F} 8,270=0.441, \mathrm{p}=0.90$ ).

The resolution of autoradiography is significantly better than that of in vivo PET. Due to the lower SUV glu $_{\text {in }}$ Cry2 ${ }^{-/}$mice, which reached trend-level significance $(p=0.054)$, we carried out ex vivo autoradiography after the last PET imaging to investigate if glucose analog $\left[{ }^{18} \mathrm{~F}\right] \mathrm{FDG}$ SUV differ after cold stress in specific brain regions of the $\mathrm{Cry}^{-/ \text {- }}$ mice (Figure 5). 2-way ANOVA taking into account the 25 studied brain regions of interest revealed significant main effects for the genotype $(F 1,199=60.87, p=3.37 E-13)$ and brain region (F24,199=10.49, $\mathrm{p}=3.76 \mathrm{E}-24)$. The interaction was not significant (genotype*brain region $\mathrm{F} 24,199=0.39$, $\mathrm{p}=0.996)$, because Cry2 ${ }^{-1-}$ mice had lower SUV than Cry2 ${ }^{++}$mice in all studied brain regions. The SUV of glucose analog $\left[{ }^{18} \mathrm{~F}\right] \mathrm{FDG}$ was lower in $\mathrm{Cry}^{-{ }^{--}}$compared to $\mathrm{Cry} 2^{++}$mice in the ventro-medial hypothalamus $[t(199)=2.59, p=0.010]$, suprachiasmatic nuclei $[t(199)=2.25, p=0.026]$, ventral tegmental area [t(199) $=3.44$, $\mathrm{p}=0.0007]$, anterior $[\mathrm{t}(199)=2.04, \mathrm{p}=0.042]$ and medial striatum $[\mathrm{t}(199)=2.04, \mathrm{p}=0.042]$, substantia nigra $[t(199)=2.06, p=0.041]$, and habenula $[t(199)=2.25, p=0.025]$ as shown by post hoc $t$-tests (Figure 5 ). None of these findings survive multiple testing correction for 25 tests ( $p<0.002$ for Bonferroni correction), and therefore, we report nominal p-values in Figure 5.

We next investigated whether the metabolic differences measured as brain glucose uptake were reflected on the gene expression levels of metabolic (Dio2, Pgcla) or depression-related (Bdnf, Dusp1, Gsk3b) genes in the brain and BAT of Cry2 $2^{--}$and Cry2 $2^{H+}$ mice at room temperature and after cold stress (Figure 6 and Supplemental Figure 2). In addition, we investigated the expression levels of Cry2 ortholog Cry1. In female Cry2 $^{-{ }^{-}}$and Cry2 ${ }^{H+}$ mice, total Bdnf and Bdnf exon 4 expression levels were lower after cold stress than at room temperature in the hippocampus. In males, similar effect of lower expression after cold stress vs. 
room temperature was seen for total Bdnf expression in the Cry2 ${ }^{H+}$ mice, and for Bdnf exon 4 in Cry2 $2^{--}$ mice. Dio2 was strongly upregulated by cold stress in both $\mathrm{Cry}^{-{ }^{--}}$and $\mathrm{Cry} 2^{++}$mice. Furthermore, Pgcla was expressed at higher levels in female $\mathrm{Cry}_{2}{ }^{-/-}$compared to $\mathrm{Cry}^{+1+}$ mice after cold stress. Interestingly, Cry1 was expressed at higher levels in male Cry2 $2^{-1-}$ compared to Cry2 ${ }^{H+}$ mice after cold stress in the hippocampus and cortex, and at room temperature in the cortex. In females, Cryl was expressed at higher levels in Cry2 ${ }^{-/}$ compared to $\mathrm{Cry} 2^{H+}$ mice after cold stress. None of the findings survived Bonferroni multiple testing correction (significance threshold $p \varangle 0.0003$ ) and therefore, we report nominal $p$-values in Figure 6.

\section{DISCUSSION}

We used multiple approaches to investigate the role of Cry2 in anxiety and depression-like behaviors in mice. We carried out a comprehensive battery of behavioral tests in $\mathrm{Cry}^{-{ }^{--}}$and $\mathrm{Cry}^{\mathrm{H+}}$ mice and investigated their brain glucose uptake both at room temperature and after cold stress. Furthermore, we investigated gene expression levels of key depression-associated and metabolic genes in both brain and BAT.

We demonstrated that Cry2 ${ }^{--}$mice had delayed onset of the first dark period activity by about two hours and slightly diminished saccharin preference, a measure of anhedonia, compared to $\mathrm{Cry} 2^{+1+}$ mice. Both of these findings are indicative of greater depressive-like behavior, although small reductions of saccharin preference (1.2-2.2\% observed here) may not have biological significance. In contrast, Cry2 ${ }^{-/-}$mice had shorter immobility time in the forced swim test, which speaks against depressive-like behavior. However, the result from the forced swim test reflects psychomotor activity, and anhedonia together with despair (longer immobility times) or reactivity (shorter immobility times) agrees with data from clinical practice. Patients with depressive disorder may have either melancholic or atypical features, respectively, or a mixture of them. Cry2 deficient mice have been considered as models for the human behavioral trait of preference to the daily activities in the evening hours ${ }^{24}$ that is known to associate with a range of health hazards including heightened odds for depressive disorders ${ }^{33}$. Furthermore, dysfunction of the circadian clocks has been associated with mood disorders ${ }^{34,35}$. It is not known, however, whether they have a causal 
effect on the etiology or whether this dysfunction reflects reactive changes later during the pathogenesis ${ }^{36}$. In humans, genetic variants of CRY2, however, are associated with mood disorders at large, including depressive disorders with the seasonal pattern or seasonal affective disorder ${ }^{37}$, chronic depressive disorder or dysthymia ${ }^{38}$, bipolar disorders with rapid cycling ${ }^{39}$, and chronicity of depressive symptoms in patients with major depressive or bipolar disorders ${ }^{40}$. A mathematical modeling for one of the key depressionassociated genetic variants of CRY2 suggests that it results in increased transcription of CRY2, leading to a decrease in winter amplitude of circadian gene expression by at least 30\% from summer amplitude, thus indicative of winter seasonal affective disorder which is characterized by atypical features during its recurrent depressive episodes ${ }^{41}$. Thus, Cry2 may influence mood through several mechanisms that may be connected or independent from each other.

After cold exposure, Cry2 ${ }^{-/-}$mice had a trend for lower glucose uptake in the posterior brain in general compared with Cry2 ${ }^{H+}$ mice, and subsequent autoradiography analysis with higher spatial resolution indicated that this finding was due to lower glucose uptake specifically in the suprachiasmatic nucleus, ventral tegmental area, ventro-medial hypothalamus, and habenula. These brain regions have previously been associated with depressive-like behavior and circadian regulation. Of specific interest is the suprachiasmatic nucleus which harbors the central clock. The involvement of the ventral tegmental area and the mesolimbic dopamine pathways in mood regulation is well documented ${ }^{42}$. For example, depressive-like behavior has been associated with long-term constant darkness in rats, which increases immobility in the forced swim test and results in larger number of apoptotic ventral tegmental area dopaminergic neurons ${ }^{43}$. Dysfunction of the mesolimbic dopamine system has also been associated with specific clock genes, e.g., depressive-like behavior in Per 2 mutant mice ${ }^{44}$ and with manic-like symptoms in the Rev-erb $\alpha$ mutant mice ${ }^{45}$. Also, the role of the habenula and its circuits in depression have recently become evident ${ }^{46}$. It may be that under a physiological challenge, such as exposure to cold or to constant lighting conditions, a genetic predisposition (here, Cry2 deficiency) results in smaller glucose uptakes in these brain regions, and leads to more severe and perhaps atypical depressive-like behavior, but this hypothesis needs to be specifically tested. 
At the gene expression level, we observed more significant cold stress effects than genotype effects. In the brain, Dio2 was expressed at higher levels after cold stress than at room temperature in all studied brain regions, in both genotypes, and in females and males. It catalyzes the conversion of prohormone thyroxine to the active thyroid hormone. Also Bdnf was expressed at lower levels after cold stress than at room temperature, especially in the hippocampus. Low levels of BDNF have been consistently observed in the brain and serum of patients with depression ${ }^{47}$. The most significant differences both after the exposure to cold and at room temperature between $\mathrm{Cry}_{2}{ }^{-/ \cdot}$ and $\mathrm{Cry} 2^{H+}$ mice were the higher expression levels of Cry1 in the $\mathrm{Cry}_{2}{ }^{-/-}$compared to $\mathrm{Cry} 2^{++}$mice in the hippocampus and cortex, but not in the hypothalamus. Cryl is a paralog of Cry2 and this upregulation may represent a compensatory effect. Consequently, some of the behavioral, metabolic or gene expression effects may be due to increased effect of Cryl expression in the hippocampus or cortex, rather than knockout of Cry2. Overall, the brain gene expression experiment suggested no differences in the expression levels of selected metabolic or depression-associated marker genes between $\mathrm{Cry}_{2}{ }^{-/-}$and $\mathrm{Cry} 2^{H+}$ mice. However, differences in the expression levels of other genes would likely be identified using transcriptome-wide methods.

In mice, the knockout effect of Cry2 on the activity of BAT has not been tested earlier. We did not observe differences in the BAT glucose uptake between $\mathrm{Cry}_{2}{ }^{-/-}$and $\mathrm{Cry} 2^{H+}$ mice using $\left[{ }^{18} \mathrm{~F}\right] \mathrm{FDG}$ PET. Although the classical BAT activation markers, such as the Ucp1 and Glut4, were significantly upregulated by cold exposure, there were only few differences in BAT gene expression levels of studied genes between Cry2 $\%$ and $\mathrm{Cry}^{\mathrm{H+}}$ mice. The exceptions were lower cold-induced upregulation of Dio2 expression in Cry2 ${ }^{-1-}$ males compared to $\mathrm{Cry2}^{+H+}$ males, and lower cold-induced upregulation of Pgcla expression in Cry2 ${ }^{-1-}$ females compared to $\mathrm{Cry}^{+++}$females. Interestingly, the Cryl expression levels were significantly higher in coldexposed males compared to the room temperature controls, in both $\mathrm{Cry}_{2}{ }^{--}$and $\mathrm{Cry} 2^{++}$mice. This upregulation seems to be specific to BAT because we did not observe similar temperature effects in the hypothalamus, hippocampus, or cortex. Overall, our results suggest that Cry2 $2^{-1-}$ mice have largely normal cold-induced BAT activation. The functional consequences and the effect on temperature regulation of the Cryl upregulation by cold stress remains to be determined. 
Our study has limitations, and it left open questions to be addressed in future work. One limitation of our mouse model is that it is a total knockout. Future studies of cell or tissue-specific models could help understand which aspects of CRY2 function regulate depression-like behavior. Also, we only studied gene expression at one time point and an analysis of several timepoints at different circadian times would allow assessing whether the differences are due to up- or downregulation or shifts in rhythms of expression. Especially the autoradiography and gene expression studies involved several measurements. None of the results survived Bonferroni correction for multiple testing ( $p$-value thresholds 0.002 and 0.0003 , respectively), and therefore, we report nominal p-values. This is a common issue in these kinds of studies, and to validate the results, they should be replicated in independent experiments. Finally, we have not been able to causally associate the brain metabolic differences between the $\mathrm{Cry}^{-{ }^{--}}$and $\mathrm{Cry} 2^{++}$mice with the behavioral data, and this remains to be attempted in future studies.

In conclusion, we replicated the earlier finding ${ }^{19}$ that Cry2 $2^{-1-}$ mice have delayed first activity peak after dark onset. We did not observe any anxiety-like phenotypes in Cry2 ${ }^{-/-}$mice using tests that measure approachavoidance conflict. These mice had an atypical depressive-like phenotype with slightly increased anhedonia and reduced despair behavior. In the future, it would be important to characterize this phenotype in more detail, investigating other depression-related symptoms, like sexual and social behaviors, sleep, and anhedonia with intracranial self-stimulation ${ }^{48}$. Our study extends previous work by showing with PET imaging and subsequent autoradiography, that glucose uptake is lower in specific depression-associated brain regions after cold stress, suggesting an association between reduced brain metabolic response to stress and behavior. Thus, our results concur with earlier findings that Cry2 is required for normal emotional behavior and we propose that this effect may be mediated by its influence on the brain metabolism.

\section{REFERENCES}

1. Rosbash M. The implications of multiple circadian clock origins. PLoS Biol. 2009;7(3):e62. 
2. M eijer JH, Michel S, Vansteensel MJ. Processing of daily and seasonal light information in the mammalian circadian clock. Gen Comp Endocrinol. 2007;152(2-3):159-164.

3. Evans JA, Suen TC, Callif BL, et al. Shell neurons of the master circadian clock coordinate the phase of tissue clocks throughout the brain and body. BM C Biol. 2015;13:43.

4. Ukai H, Kobayashi TJ, Nagano M, et al. M elanopsin-dependent photo-perturbation reveals desynchronization underlying the singularity of mammalian circadian clocks. Nat Cell Biol. 2007;9(11):1327-1334.

5. Dardente H, Fortier EE, M artineau V, Cermakian N. Cryptochromes impair phosphorylation of transcriptional activators in the clock: a general mechanism for circadian repression. Biochem J. 2007;402(3):525-536.

6. Ozturk N, Song SH, Ozgur S, et al. Structure and function of animal cryptochromes. Cold Spring Harb Symp Quant Biol. 2007;72:119-131.

7. Ye R, Selby CP, Chiou YY, Ozkan-Dagliyan I, Gaddameedhi S, Sancar A. Dual modes of CLOCK:BM AL1 inhibition mediated by Cryptochrome and Period proteins in the mammalian circadian clock. Genes Dev. 2014;28(18):1989-1998.

8. Ye R, Selby CP, Ozturk N, Annayev Y, Sancar A. Biochemical analysis of the canonical model for the mammalian circadian clock. J Biol Chem. 2011;286(29):25891-25902.

9. Zhang EE, Liu AC, Hirota T, et al. A genome-wide RNAi screen for modifiers of the circadian clock in human cells. Cell. 2009;139(1):199-210.

10. Sato TK, Yamada RG, Ukai H, et al. Feedback repression is required for mammalian circadian clock function. Nat Genet. 2006;38(3):312-319.

11. Ukai-Tadenuma M, Yamada RG, Xu H, Ripperger JA, Liu AC, Ueda HR. Delay in feedback repression by cryptochrome 1 is required for circadian clock function. Cell. 2011;144(2):268-281.

12. Ono D, Honma S, Honma K. Cryptochromes are critical for the development of coherent circadian rhythms in the mouse suprachiasmatic nucleus. Nat Commun. 2013;4:1666. 
13. Griesauer I, Diao W, Ronovsky M, et al. Circadian abnormalities in a mouse model of high trait anxiety and depression. Ann Med. 2014;46(3):148-154.

14. De Bundel D, Gangarossa G, Biever A, Bonnefont X, Valjent E. Cognitive dysfunction, elevated anxiety, and reduced cocaine response in circadian clock-deficient cryptochrome knockout mice. Front Behav Neurosci. 2013;7:152.

15. Savalli G, Diao W, Berger S, Ronovsky M, Partonen T, Pollak DD. Anhedonic behavior in cryptochrome 2-deficient mice is paralleled by altered diurnal patterns of amygdala gene expression. Amino acids. 2015;47(7):1367-1377.

16. Huhne A, Volkmann P, Stephan M, Rossner M, Landgraf D. An in-depth neurobehavioral characterization shows anxiety-like traits, impaired habituation behavior, and restlessness in male Cryptochrome-deficient mice. Genes Brain Behav. 2020:e12661.

17. Schnell A, Sandrelli F, Ranc V, et al. Mice lacking circadian clock components display different mood-related behaviors and do not respond uniformly to chronic lithium treatment. Chronobiol Int. 2015;32(8):1075-1089.

18. van der Horst GT, M uijtjens M, Kobayashi K, et al. M ammalian Cry1 and Cry2 are essential for maintenance of circadian rhythms. Nature. 1999;398(6728):627-630.

19. Thresher RJ, Vitaterna M H, M iyamoto $Y$, et al. Role of mouse cryptochrome blue-light photoreceptor in circadian photoresponses. Science. 1998;282(5393):1490-1494.

20. Griffin EA, Jr., Staknis D, Weitz CJ. Light-independent role of CRY1 and CRY2 in the mammalian circadian clock. Science. 1999;286(5440):768-771.

21. Vitaterna $\mathrm{MH}$, Selby $\mathrm{CP}$, Todo $\mathrm{T}$, et al. Differential regulation of mammalian period genes and circadian rhythmicity by cryptochromes 1 and 2. Proc Natl Acad Sci U SA. 1999;96(21):1211412119.

22. Nagashima K, Matsue K, Konishi M, et al. The involvement of Cryl and Cry2 genes in the regulation of the circadian body temperature rhythm in mice. Am J Physiol Regul Integr Comp physiol. 2005;288(1):R329-335. 
23. Fuller CA, Sulzman FM, Moore-Ede MC. Thermoregulation is impaired in an environment without circadian time cues. Science. 1978;199(4330):794-796.

24. Destici E, Jacobs EH, Tamanini F, Loos M, van der Horst GT, Oklejewicz M. Altered phaserelationship between peripheral oscillators and environmental time in Cry1 or Cry2 deficient mouse models for early and late chronotypes. PLoS One. 2013;8(12):e83602.

25. Ricquier D, Kader JC. M itochondrial protein alteration in active brown fat: a soidum dodecyl sulfatepolyacrylamide gel electrophoretic study. Biochem Biophys Res Comm. 1976;73(3):577-583.

26. Diaz MB, Herzig S, Vegiopoulos A. Thermogenic adipocytes: from cells to physiology and medicine. Metab Clin Exp. 2014;63(10):1238-1249.

27. Himms-Hagen J. Nonshivering thermogenesis. Brain Res Bull. 1984;12(2):151-160.

28. Ootsuka Y, de Menezes RC, Zaretsky DV, et al. Brown adipose tissue thermogenesis heats brain and body as part of the brain-coordinated ultradian basic rest-activity cycle. Neuroscience. 2009;164(2):849-861.

29. Blessing W, M ohammed M, Ootsuka Y. Brown adipose tissue thermogenesis, the basic rest-activity cycle, meal initiation, and bodily homeostasis in rats. Physiol Behav. 2013;121:61-69.

30. Yang YL, Shen ZL, Tang Y, Wang N, Sun B. [Simultaneous telemetric analyzing of the temporal relationship for the changes of the circadian rhythms of brown adipose tissue thermogenesis and core temperature in the rat]. Chinese journal of applied physiology. 2011;27(3):348-352.

31. Zvonic S, Ptitsyn AA, Conrad SA, et al. Characterization of peripheral circadian clocks in adipose tissues. Diabetes. 2006;55(4):962-970.

32. Sacher J, Neumann J, Funfstuck T, Soliman A, Villringer A, Schroeter ML. Mapping the depressed brain: a meta-analysis of structural and functional alterations in major depressive disorder. J Affect Disord. 2012;140(2):142-148.

33. Merikanto I, Kronholm E, Peltonen M, Laatikainen T, Vartiainen E, Partonen T. Circadian preference links to depression in general adult population. J Affect Disord. 2015;188:143-148.

34. Baron KG, Reid KJ. Circadian misalignment and health. Int Rev Psychiatry. 2014;26(2):139-154. 
35. Logan RW, Edgar N, Gillman AG, Hoffman D, Zhu X, M cClung CA. Chronic Stress Induces Brain Region-Specific Alterations of M olecular Rhythms that Correlate with Depression-like Behavior in M ice. Biol Psychiatry. 2015;78(4):249-258.

36. McCarthy MJ, Welsh DK. Cellular circadian clocks in mood disorders. J Biol Rhythms. 2012;27(5):339-352.

37. Lavebratt C, Sjoholm LK, Soronen P, et al. CRY2 is associated with depression. PLoS One. 2010;5(2):e9407.

38. Kovanen L, Kaunisto M, Donner K, Saarikoski ST, Partonen T. CRY2 genetic variants associate with dysthymia. PLoS One. 2013;8(8):e71450.

39. Sjoholm LK, Backlund L, Cheteh EH, et al. CRY2 is associated with rapid cycling in bipolar disorder patients. PLoS One. 2010;5(9):e12632.

40. Fiedorowicz JG, Coryell WH, Akhter A, Ellingrod VL. Chryptochrome 2 variants, chronicity, and seasonality of mood disorders. Psychiatr Genet. 2012;22(6):305-306.

41. Liberman AR, Halitjaha L, Ay A, Ingram KK. M odeling Strengthens M olecular Link between Circadian Polymorphisms and M ajor M ood Disorders. J Bioll Rhythms. 2018;33(3):318-336.

42. Han MH, Nestler EJ. Neural Substrates of Depression and Resilience. Neurotherapeutics. 2017;14(3):677-686.

43. Gonzalez M M , Aston-Jones G. Light deprivation damages monoamine neurons and produces a depressive behavioral phenotype in rats. Proc Natl Acad Sci U SA. 2008;105(12):4898-4903.

44. Hampp G, Ripperger JA, Houben T, et al. Regulation of monoamine oxidase A by circadian-clock components implies clock influence on mood. Curr Biol. 2008;18(9):678-683.

45. Chung S, Lee EJ, Yun S, et al. Impact of circadian nuclear receptor REV-ERBalpha on midbrain dopamine production and mood regulation. Cell. 2014;157(4):858-868.

46. Hu H, Cui Y, Yang Y. Circuits and functions of the lateral habenula in health and in disease. Nat Rev Neurosci. 2020;21(5):277-295. 
47. Castren E, Kojima M. Brain-derived neurotrophic factor in mood disorders and antidepressant treatments. Neurobiol Dis. 2017;97(Pt B):119-126.

48. Cryan JF, Holmes A. The ascent of mouse: advances in modelling human depression and anxiety. Nat Rev Drug Discov. 2005;4(9):775-790.

\section{ACKNOWLEDGEMENTS}

Supported by Sigrid Jusélius Foundation and University of Helsinki (to I.H.). We thank Matti Jauhiainen for carrying out the FFA measurements, and Emma Roivainen for analyzing the BAT density data. M ouse behavioral phenotyping facility at the University of Helsinki is supported by Biocenter Finland and Helsinki Institute of Life Science. PET/CT studies were conducted within the Finnish Centre of Excellence in Cardiovascular and M etabolic Disease supported by the Academy of Finland, University of Turku, Turku University Hospital and Åbo Akademi University, and further supported by grants from the Academy of Finland (\#258814) and Hospital District of Southwest Finland (\#13856).

\section{CONFLICT OF INTEREST STATEM ENT}

The authors report no conflicts of interest.

\section{DATA AVAILABIUTY STATEM ENT}

Data presented in this paper are available from the corresponding author on reasonable request. 


\section{FIGURE LEGENDS}

Figure 1. No difference in anxiety-like behavior or exploratory activity in $\mathrm{Cry2}^{-/ \cdot}$ and $\mathrm{Cry2}^{+/+}$mice. We tested $\mathrm{Cry2}^{--}(\mathrm{ko}, \mathrm{n}=24)$ and $\mathrm{Cry}_{2}{ }^{++}(\mathrm{wt}, \mathrm{n}=27)$ mice in several tests that measure anxiety-like behavior and locomotor activity. (A) Percent time spent in the center zone of the open field. (B) Distance traveled in the open field. (C) Percent time spent in the light zone in the light-dark box test. (D) Latency to the first entry to light compartment in the light-dark box test. (E) Total distance travelled during the light-dark box test. (F) Latency to feeding in the novelty suppressed feeding test. $(G)$ Weight of food eaten during 5 min in home cage. Due to no significant sex differences, male and female mice were pooled. Bar graphs show mean \pm 1 s.e.

Figure 2. Cry2 ${ }^{-/-}$mice differ from $\mathrm{Cry2}^{+/+}$mice in tests measuring depression-like behavior. (A) Cry2 $2^{-1-}$ mice (ko, $n=24)$ spent less time immobile during the last 4 minutes in the forced swim test than Cry2 $2^{++}$mice (wt, $n=27$ ), suggesting decreased despair-like behavior. (B) Cry2 $\%$ mice $(n=22)$ had significantly decreased preference to both $0.1 \%$ and $0.2 \%$ saccharin compared to Cry $2^{H+}$ mice $(n=24)$, indicating increased anhedonia. Due to no significant sex differences, male and female mice were pooled. Bar graphs show mean \pm 1 s.e.

Figure 3. Cry2 $\%$ mice have shifted circadian activity without changes in total activity. (A) No difference in

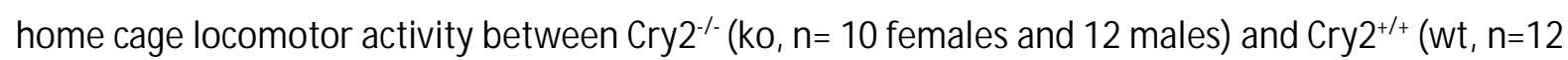
females and 13 males) mice. Both male (B) and female (C) Cry2 ${ }^{-/}$mice had delayed night activity period with the peak activity two hours later than in $\mathrm{Cry} 2^{++}$mice. Bar and line graphs show mean averaged over 5 days \pm 1 s.e.

Figure 4. Positron emission tomography with glucose analog $\left[{ }^{18} \mathrm{~F}\right] \mathrm{FDG}$ in $\mathrm{Cry2}^{-/-}$and $\mathrm{Cry2}^{+/+}$mice. To assess brain metabolic activation, we carried out positron emission tomography on female $\mathrm{Cry} 2^{-{ }^{-}}(n=10)$ and $\operatorname{Cry2}^{++}(n=7)$ mice with glucose analog [ $\left.{ }^{18} \mathrm{~F}\right] \mathrm{FDG}$. (A) Glucose consumption (SUV $\mathrm{glu}$ ) at room temperature, and (B) after 4-hour cold exposure. Bar graphs show mean \pm 1 s.e. 
Figure 5. Cry2 ${ }^{-/-}$mice have lower glucose consumption than $\mathrm{Cry}^{+/+}$mice in specific brain regions after cold stress. We carried out autoradiography directly after PET imaging and calculated standardized uptake value (SUV) of glucose analog $\left[{ }^{18} \mathrm{~F}\right] \mathrm{FDG}$ from brain regions of interest in $\mathrm{Cry}^{-{ }^{--}}(n=5)$ and $\mathrm{Cry}^{+++}(n=5)$ mice. Bar graphs show mean \pm 1 s.e.

Figure 6. Gene expression levels of metabolic and depression-associated genes in $\mathrm{Cry}^{-{ }^{--}}$and $\mathrm{Cry}^{2+/+}$ mice at room temperature (RT) and after 4-hour cold stress. Relative gene expression levels, determined by quantitative real-time PCR, in the hippocampus (HPC), hypothalamus (HT), and cortex (CX). Bar graphs show mean +1 s.e. 
(A)

Open field

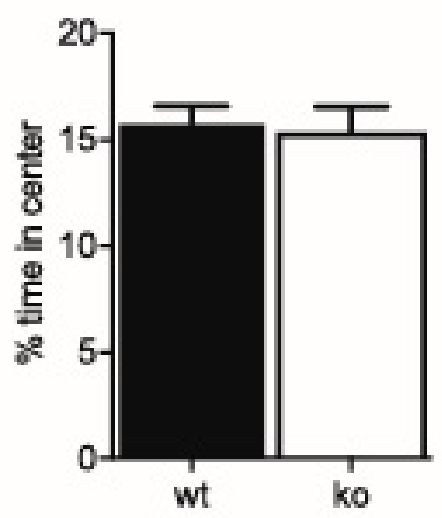

(B) Open field

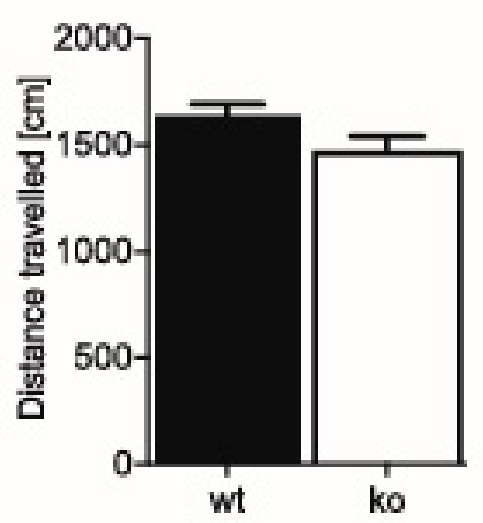

(C) Light-dark box
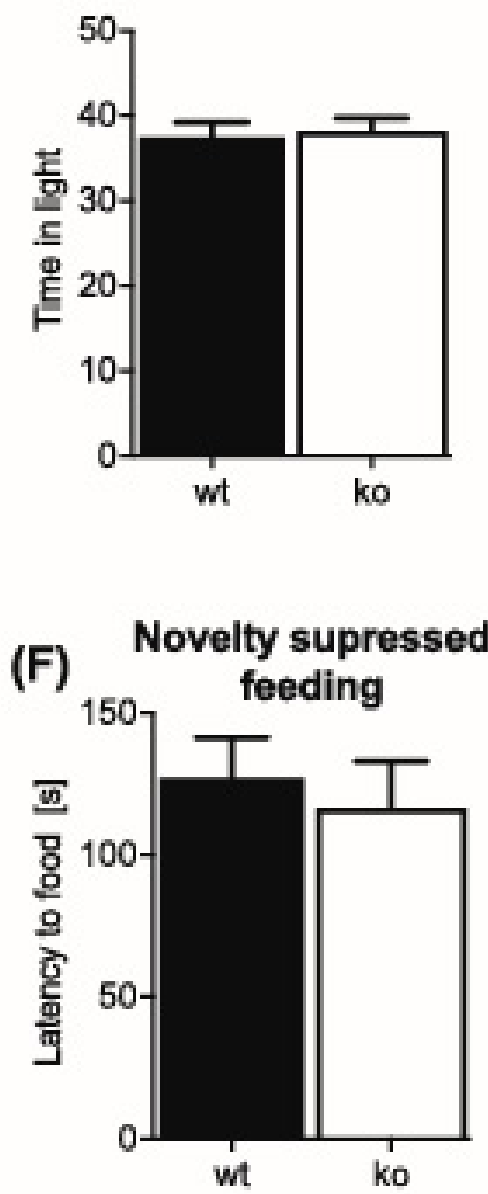

(D) Light-dark box

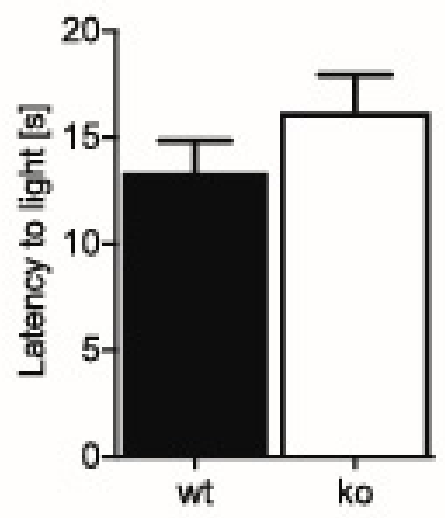

(E) Light-dark box

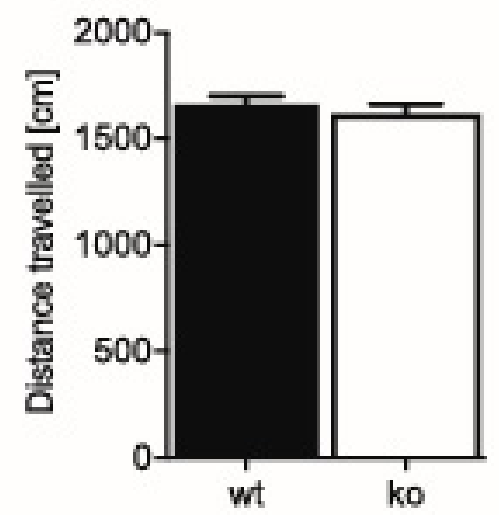

(G) Novelty supressed

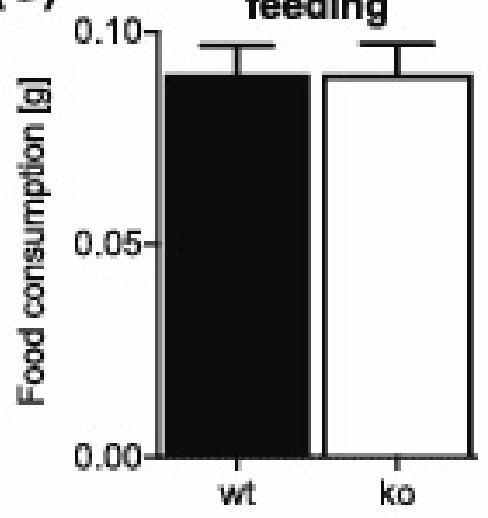


(A)

Forced swim test

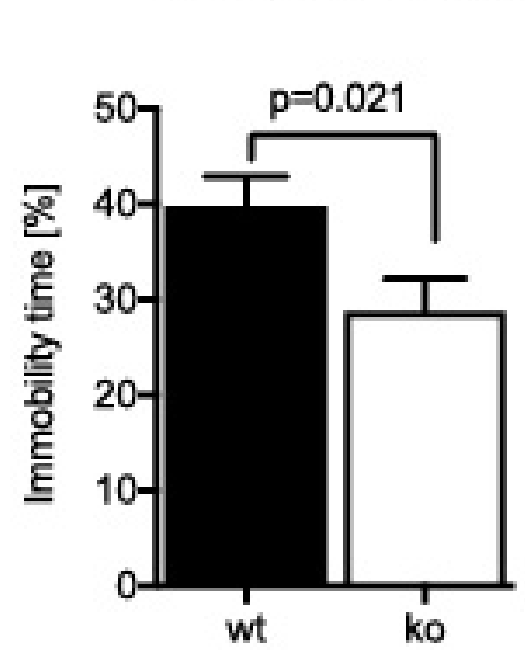

(B)

Saccharin preference

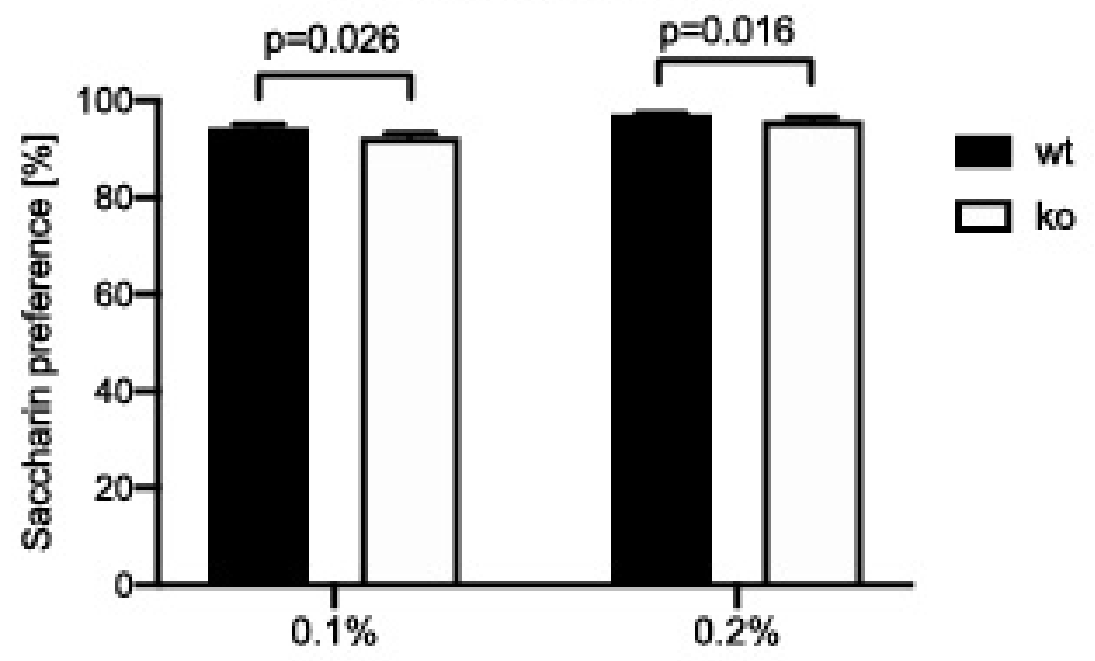




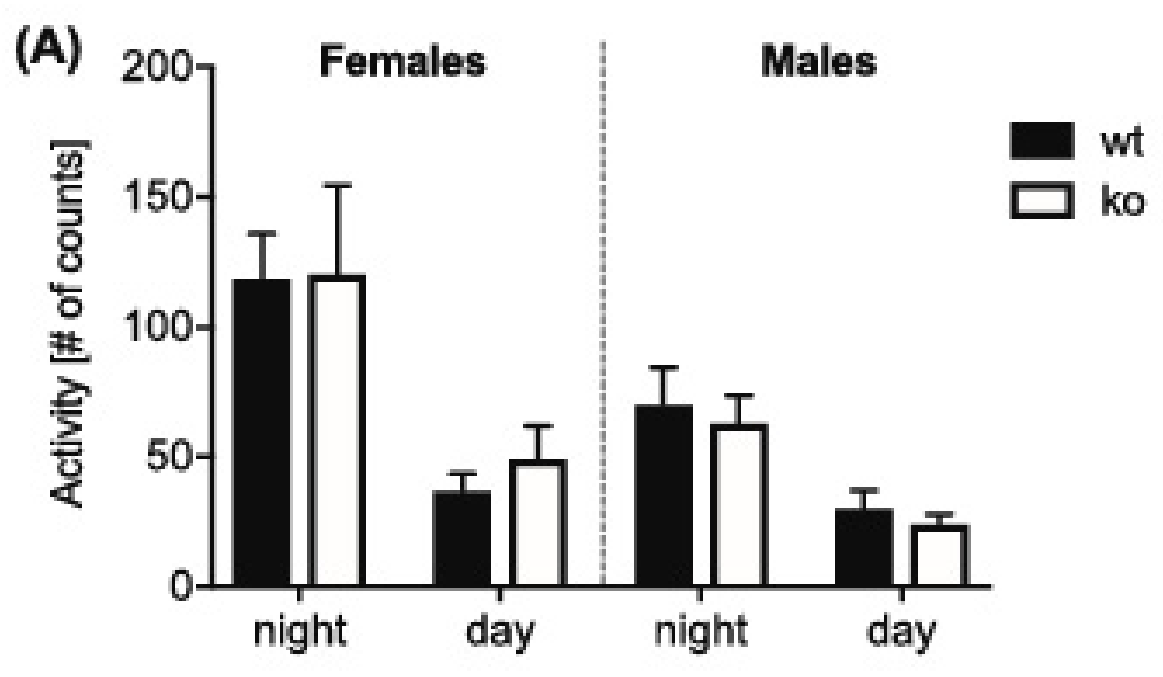

(B)

Females

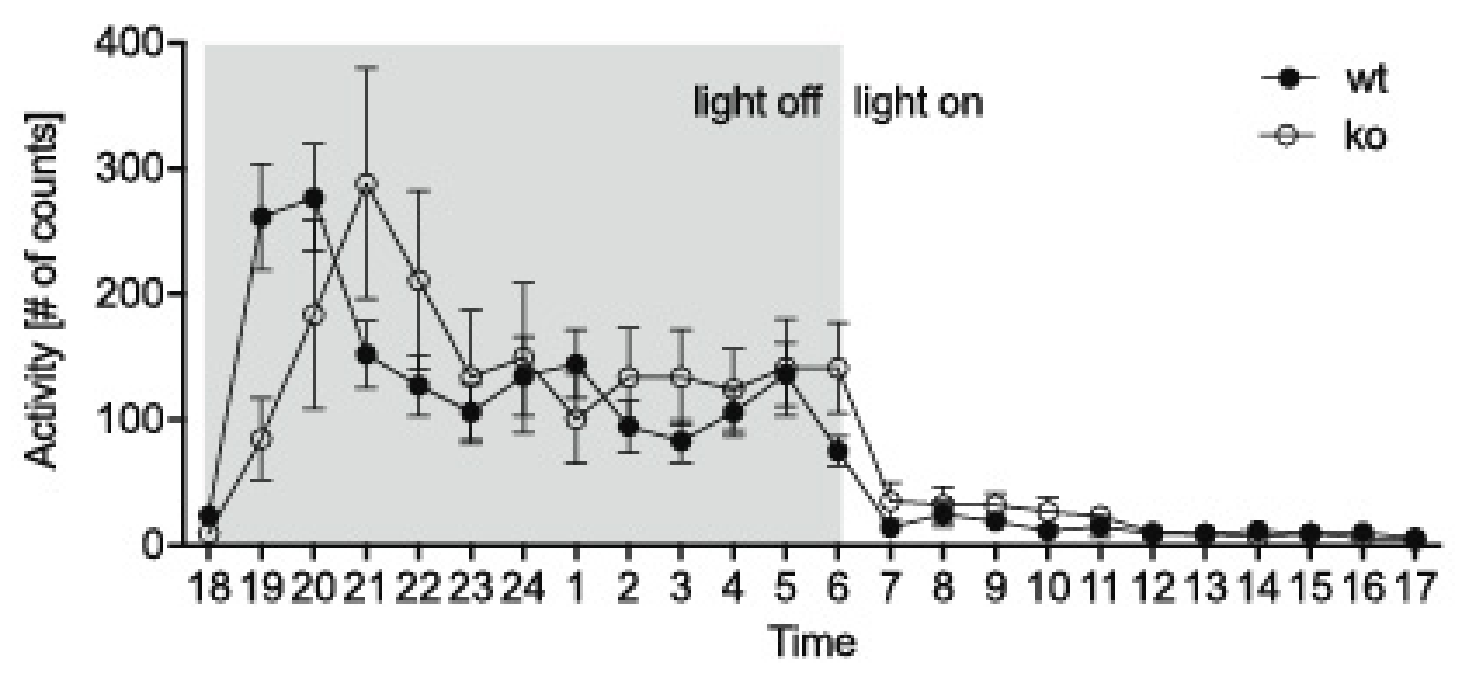

(C)

Males

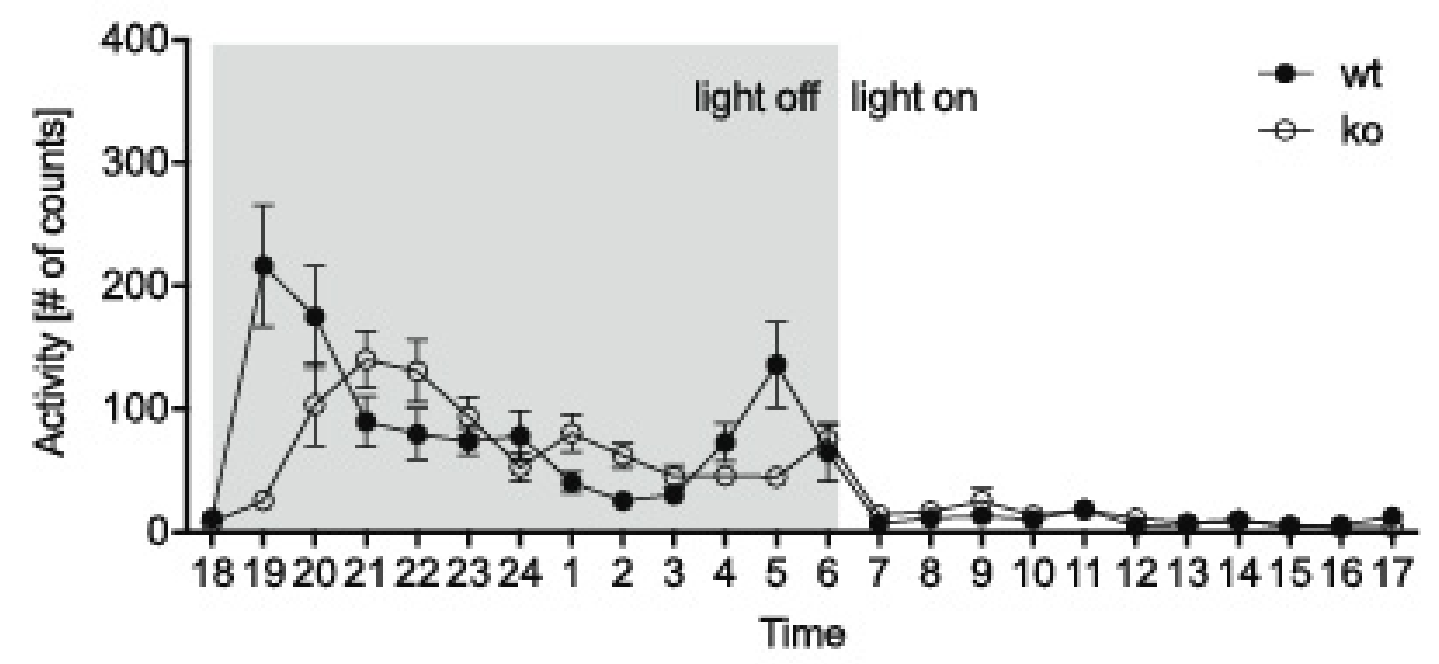




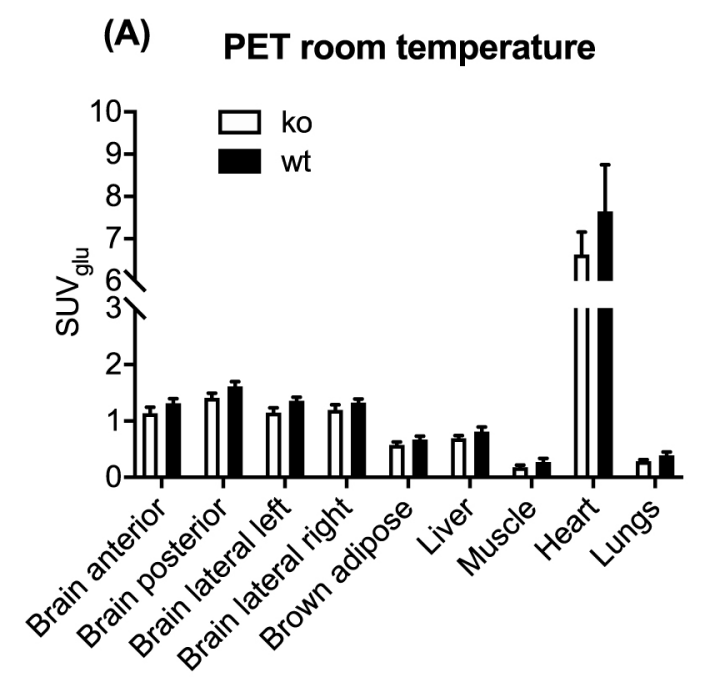

(B) PET after cold exposure

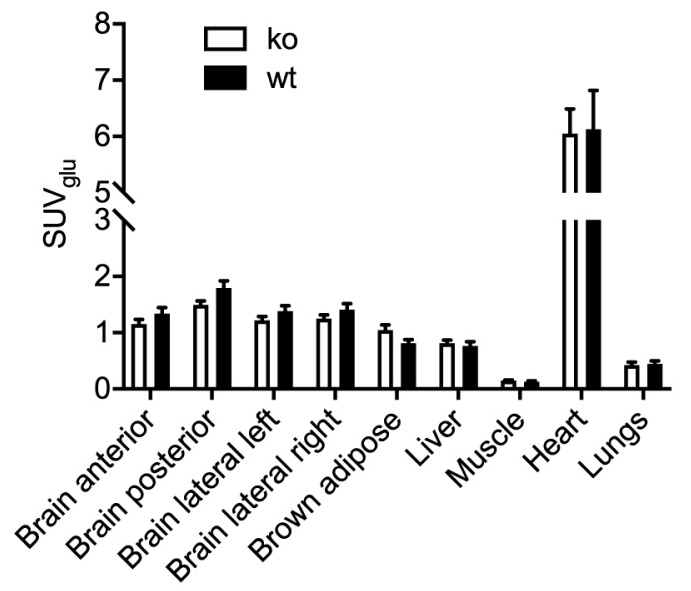




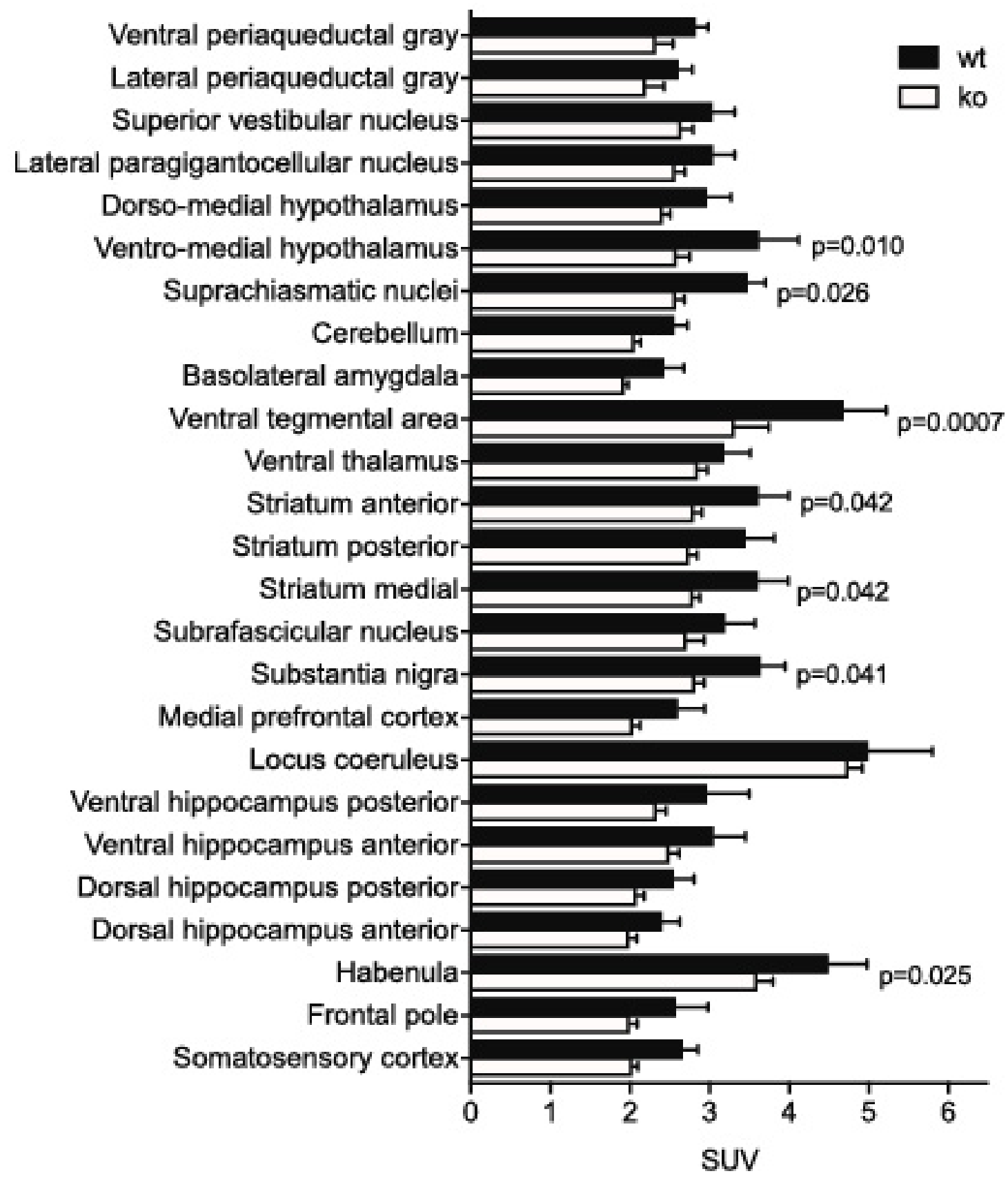



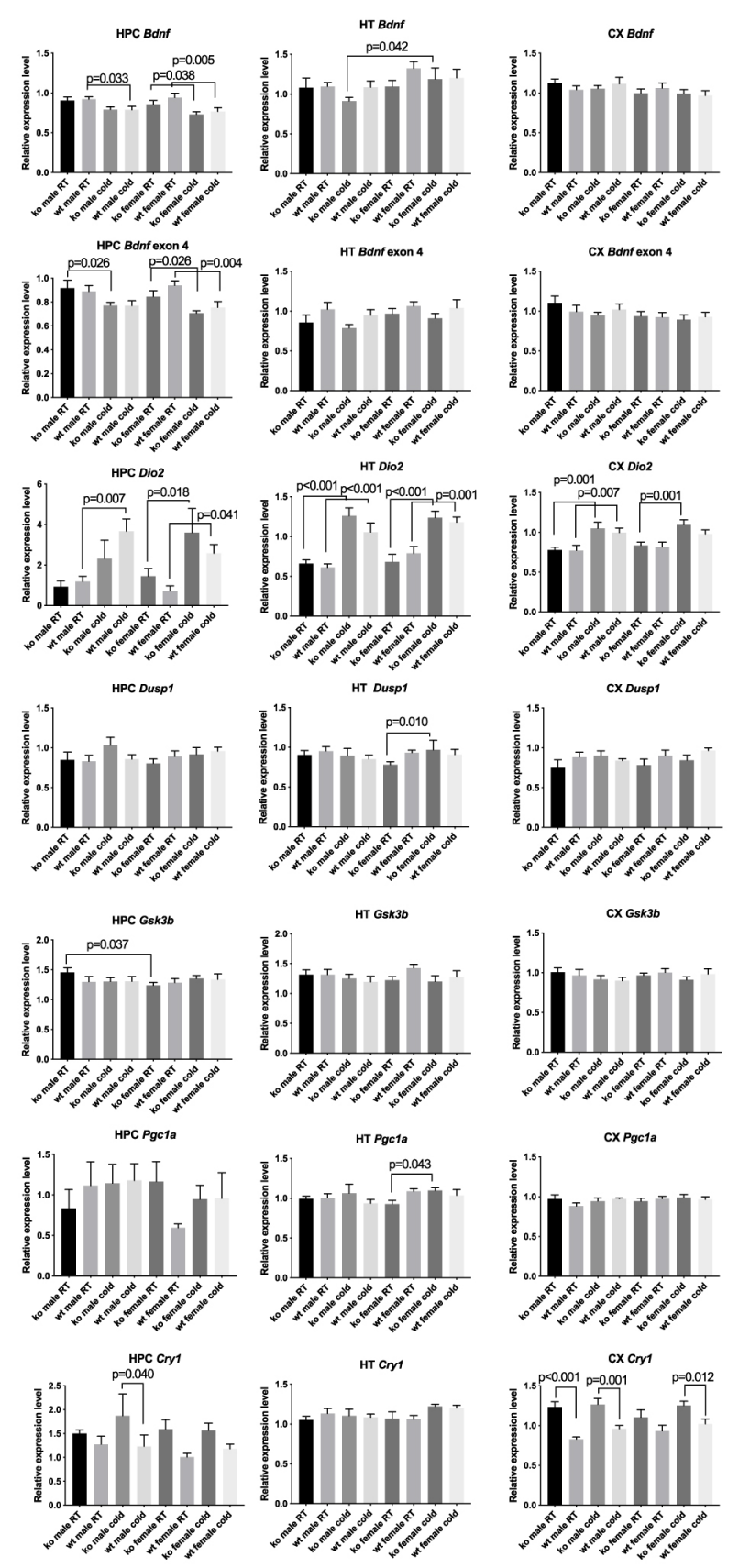
Plasma glucose levels before and after ${ }^{18}$ F-FDG PET

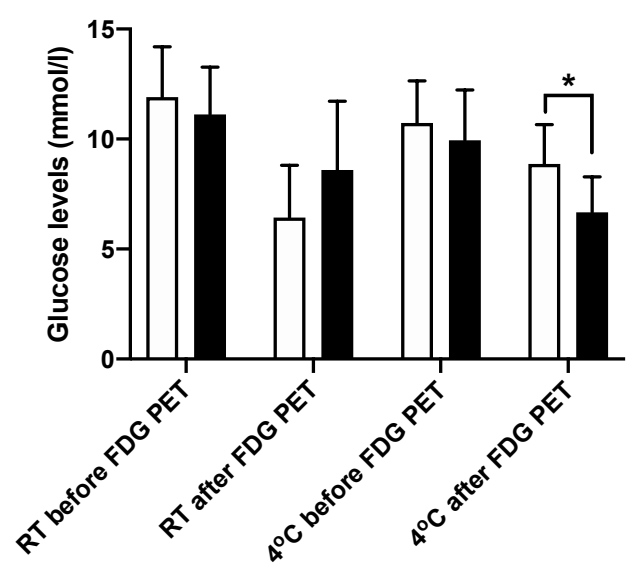

C

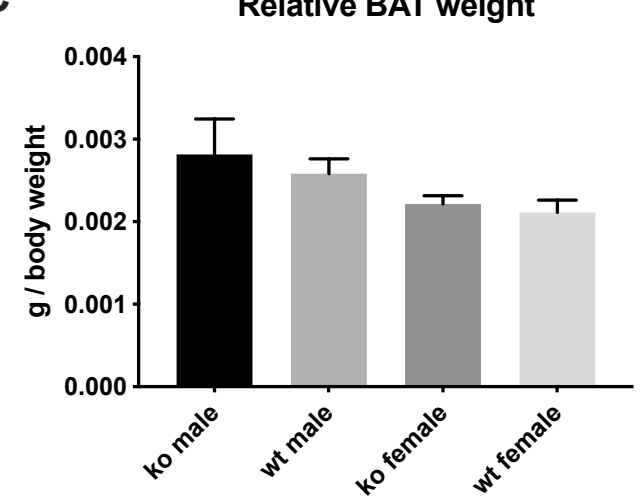

B

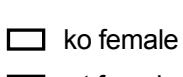

wt female

D Relative BAT density in CT

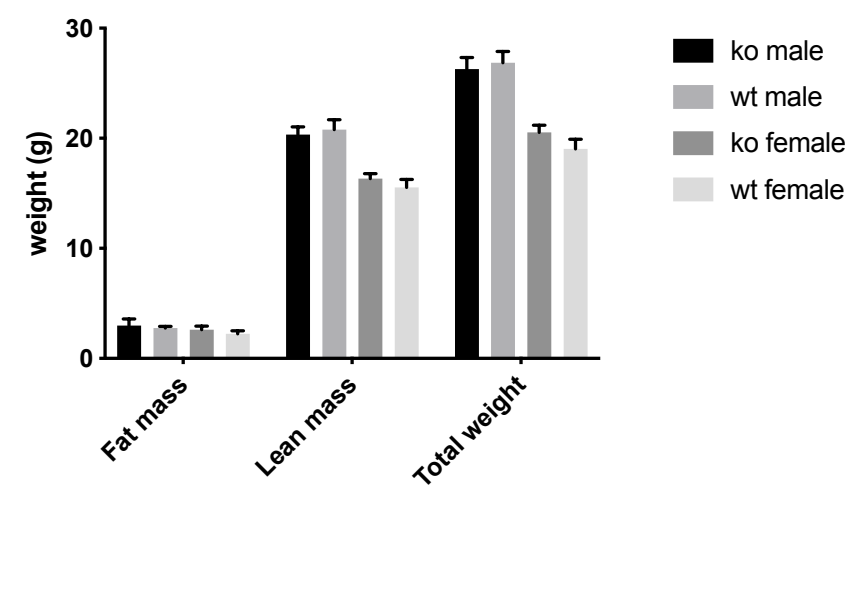

Body composition

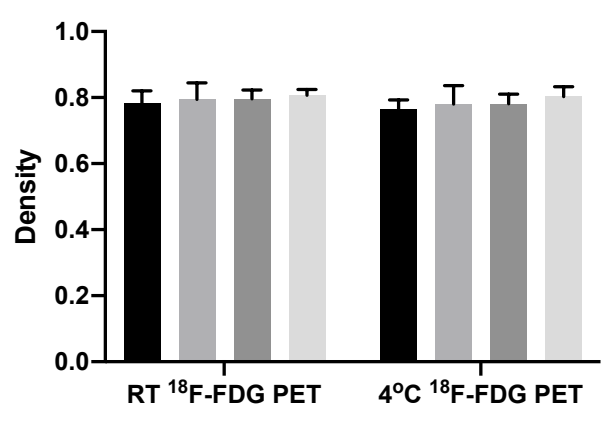

ko male

wt male

ko female

wt female

Supplemental figure 1. Plasma glucose levels, body composition and BAT weight and density of Cry2 ${ }^{-/-}$ and $\mathrm{Cry2}^{+/+}$mice. A) Plasma glucose levels measured before ${ }^{18} \mathrm{~F}$-FDG PET carried out in room temperature (RT) or after $4 \mathrm{~h}$ cold exposure at $4^{\circ} \mathrm{C}$. Knockout (ko) $\mathrm{N}=10$, wild type (wt) $\mathrm{N}=7$. B) Body composition measured by quantitative nuclear magnetic resolution whole-body composition analyzer. Ko male $\mathrm{N}=6$, wt male $\mathrm{N}=2$, ko female $\mathrm{N}=10$, wt female $\mathrm{N}=7$. C) Brown adipose tissue (BAT) weight normalized by total body weight. Ko male $\mathrm{N}=4$, wt male $\mathrm{N}=2$, ko female $\mathrm{N}=9$, wt female $\mathrm{N}=6$. D) BAT density normalized by tibia density measured by computed tomography. Ko male $\mathrm{N}=4-5$, wt male $\mathrm{N}=2$, ko female $\mathrm{N}=10$, wt female $\mathrm{N}=7$. ${ }^{*} \mathrm{p}<0.05$ in 2-way ANOVA followed by Fisher's LSD post hoc test. 
Supplemental figure 2.

BAT Ucp1

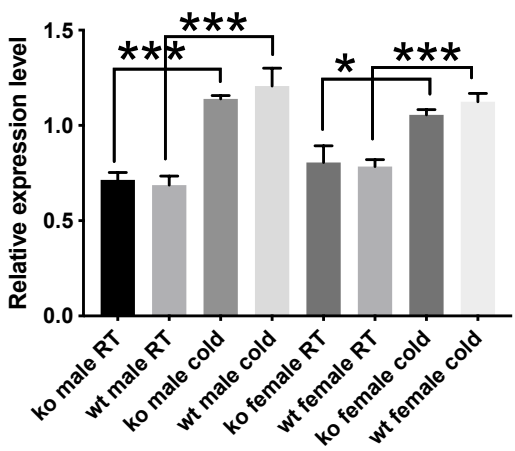

BAT Glut4

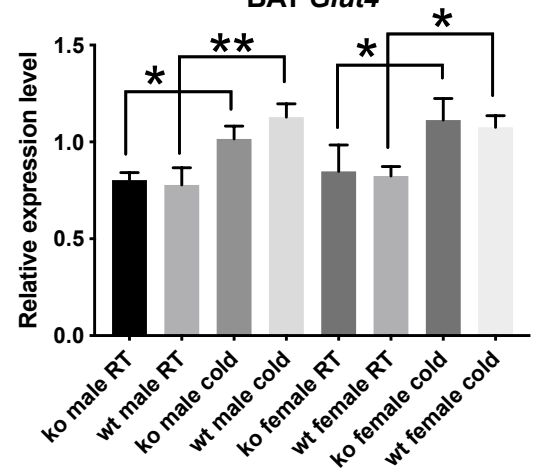

BAT Prdm16

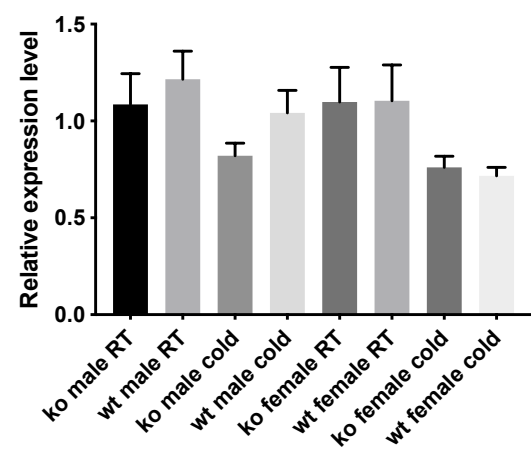

BAT HsI

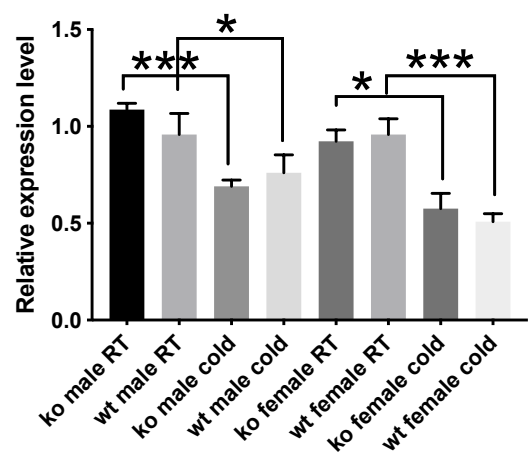

BAT Adrb3

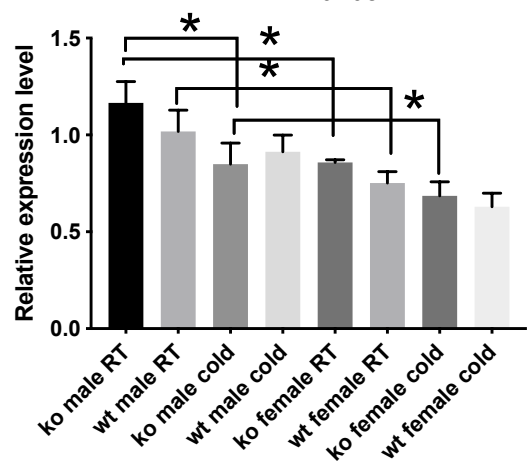

BAT Pck1

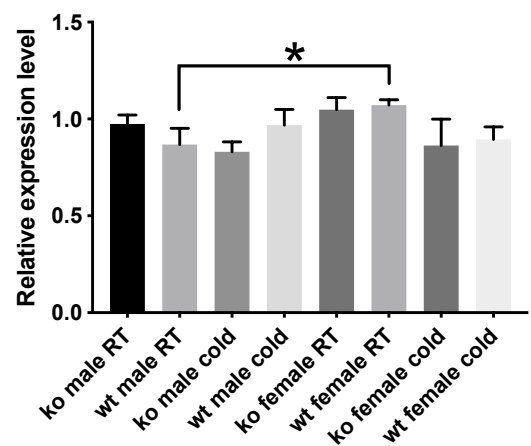

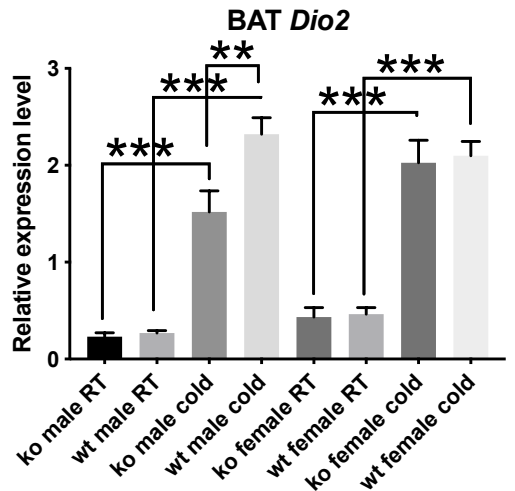

BAT Pgc1a
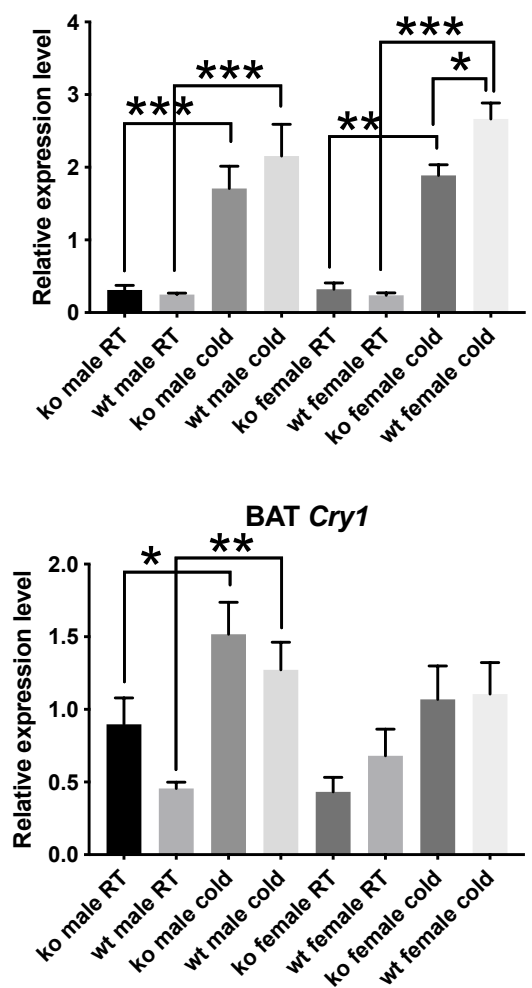

Supplemental figure 2. Gene expression levels of metabolic genes in $\mathrm{Cry}^{-/-}$and $\mathrm{Cry2}^{+/+}$mice at room temperature (RT) and after $\mathbf{4} \mathrm{h}$ cold stress. Relative gene expression levels, determined by quantitative realtime PCR, in the brown adipose tissue (BAT). Bar graphs show mean $+/-1$ s.e. Nominal $P$-values $<0.05$ from $3-$ way ANOVA followed by Fisher's LSD post hoc test. ${ }^{*} p<0.05,{ }^{* *} p<0.01,{ }^{* * *} p<0.001$. Each group $N=7$. 